\title{
Old inconsistency in electromagnetism and a way to eliminate it
}

\author{
Valery Petuschak ${ }^{1}$ \\ ${ }^{1}$ VM Glushkov Institute of Cybernetics, Kiev, 03187, Ukraine \\ E-mail: v.petuschak@gmail.com
}

\section{ABSTRACT}

Classical electromagnetic theory uses axial pseudo-vectors to describe magnetic interactions. It is impossible to explain adequately magnetic interaction at the micro level (elements of conductors and the magnetic interactions of charges) by axial vectors. As a result, the correct form of interactions in classical electrodynamics is only an integral one. The differential formulas for magnetic interactions violate the third Newton's law.

In the paper, we use polar vectors (real physical vectors) to describe magnetic interactions. On this way, we show that the real physical magnetic field, in contrast to the solenoidal field of the axial vector magnetic induction $\boldsymbol{B}$, has two components: a potential field with nonvanishing divergence and a solenoidal field with vanishing divergence. These two fields act separately and independently and have different models of interactions.

Doing so, we can write differential form for the Ampere's law obtaining correct formula for the magnetic interactions and adequate interpretation of the Biot-Savart law.

\section{Key words}

Electromagnetism, magnetic force, Ampere's law, Whittaker's formula, two-component magnetic field, consistent theory.

PACS number: 40.

\section{INTRODUCTION}

Faraday, Ampere, Biot and other scientists started to study electromagnetic interactions nearly two hundred years ago. In 1826, Ampere published his "Electrodynamics" [2] with a subtitle "Theory of electrodynamic phenomena deduced exclusively from experiments". Ampere described his own experiments, cited Faraday experiments, and suggested the formula for conductors elements interaction following from these experiments. However, after Ampere's death, physicists adopted Grassmann formula, known nowadays as Ampere's law. Almost two hundred years later, the electrodynamics cannot explain Ampere's and Faraday's experiments demonstrating axial magnetic interaction of current-carrying conductors: the Biot-Savart law controverts any probability of such interactions. The direction of the magnetic forces in these experiments is also surprising: the conductors repel, having the same directions of currents.

Recent ten years radio-amateurs use radio communication with the so called $\mathrm{E}-\mathrm{H}$ aerials, that emit and receive radio waves in the axial direction of antenna conductors. The existence of this radio communication cannot be explained theoretically.

How was this situation created and why? Why do we know now dozens of the so called "paradoxical experiments" not explained by the modern electrodynamics? Why do scientific articles and books contain examples of violating the equality of action and counteraction forces in magnetic interactions of conductors, that is, violations of the Newton's third law (I.Tamm [3] p.223).

In what follows, we analyze the causes of these problems in electromagnetism and the correctness of the modern magnetic field theory.

\section{Models of electromagnetic interactions}

Two hundred years ago the possibilities to study electromagnetic interactions were poor. Therefore, model thinking of these interactions played an important role.

Ampere's model recognized interactions by central forces only: "the laws (of electromagnetic phenomena) must be the only basis when deducing a formula determining the elementary forces, whose direction coincides with a straight line connecting two interacting material points" [2]. Oersted pictured the interaction model in a different way [4]. Ampere cites him [2]: "the observations done (interaction of a conductor with current and a magnet) lead to the conclusion that this conflict generates a vortex around the wire." Other physicists, for example Biot, believed that the interaction is caused by a couple of non-central parallel forces, namely, the force of action and the force of counteraction, applied to the source of forces in compliance with the third Newton's law. Such a couple of forces generates a torque in the line with Oersted's assumption.

Ampere proved his model by the experiments. In [2], he described the experiment when a metal frame with current ABCDE moves in mercury in the direction of electric current due to forces of repulsion acting in axial direction of conductors $A B$ and $E D$ (Fig.1).

Ampere cites Faraday's experiment giving the same result [2]: "the repulsion force acts on a very thin conductor suspended vertically with the end immersed into mercury". This experiment is described by Faraday [5]. The experiment was done both with a single conductor and a frame with two parallel vertical conductors with ends immersed into 
mercury. The two conductors repel from mercury surface, although the directions of current in these conductors were opposite. Faraday described this as follows: "when I closed the circuit ... with a galvanic element, the conductors rose

one inch above the mercury surface. They dropped off again when the circuit was disconnected".

These experiments proved the existence of axial magnetic forces between current-carrying conductors. The most surprising was the direction of magnetic forces: the conductors repelled despite that the currents in the conductor and its continuation in mercury had the same directions.

The correctness of experiments is undeniable. Ampere's experiment with the frame was not left out of physicists' attention. Maxwell recalled it in [6]. He explained the experiment basing on equal results that both Ampere's and Grassmann's formulas give for closed circuits.

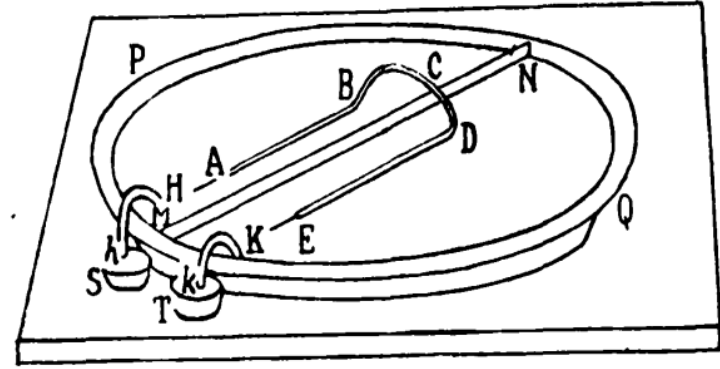

Fig. 1. $P Q$ is a glass vessel divided into two chambers with mercury by the screen $M N$. $A B C D E$ is an isolated copper conductor floating in mercury. Non-isolated ends of the conductor, $A$ and $E$, are in contact with mercury. $S$ and $T$ are contacts with a potential difference between them.

The correctness of experiments is undeniable. Ampere's experiment with the frame was not left out of physicists' attention. Maxwell recalled it in [6]. He explained the experiment basing on equal results that both Ampere's and Grassmann's formulas give for closed circuits.

How shall Grassman's formula explain the experiment with the frame basing on Maxwell's standpoint? According to this formula, the interaction is realized by forces orthogonal to conductors axis. The only possible option is the motion of the BCDE framework by the "unbalanced" actions of the BCD conductor on the conductors AB and DE. The conductors are fastened, so the frame is driven by its own magnetic field.

R.Sigalov [7] developed the idea of a conductor motion by its own magnetic field, giving dozens of experiments, many of which he performed himself. In the experiments, conductors with current were moved by the "unbalanced forces" of their own magnetic field. Below we consider if is it possible moving of the conductor by its own magnetic field.

In 1980, Pappas [8] gave a self-made version of the experiment with the frame (Fig. 2). The frame was made from a lightweight aluminum tube of $2 \mathrm{~mm}$ in diameter, weighing $38.2 \mathrm{~g}$. The length of the frame is $2 \mathrm{~m}$, the width is $1.5 \mathrm{~m}$. The frame was suspended on thin wires not preventing it to move horizontally. The ends of the frame touch the mercury surface in two small cups B and $E$ with potential difference between them. When current flew through the circuit, the frame moved forward at a speed of $2 \mathrm{~cm} / \mathrm{s}$ while its ends touched the mercury surface.

Pappas does not agree with Maxwel: "Maxwell's statement about the identity of both laws makes sense only when $\boldsymbol{d}_{\boldsymbol{1}}$ is not a part of the contour $\boldsymbol{I}_{2}$. When $\boldsymbol{d} \boldsymbol{I}_{1}$ is a part of contour $\boldsymbol{I}_{2}$, Maxwell's statement is not true.

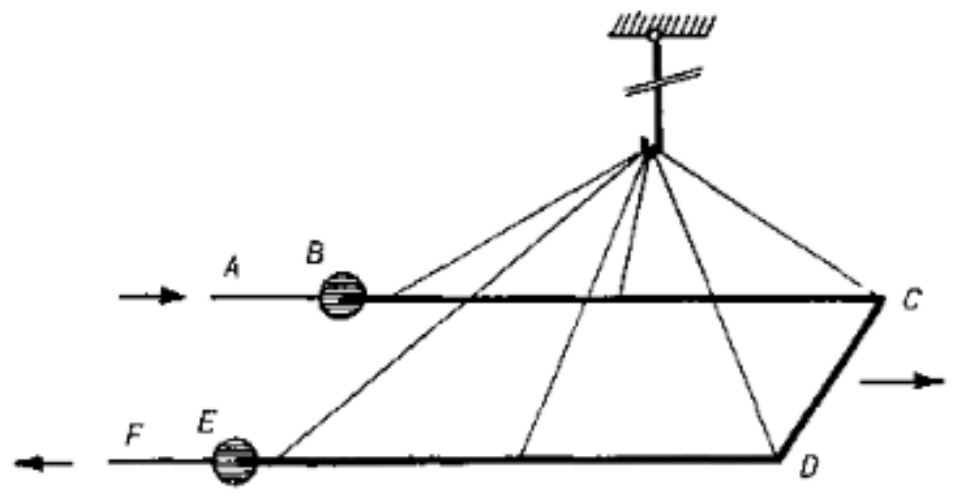

Fig. 2 Pappas experiment with frame.

The formula Ampere proposed for interaction of conductors elements in today representation is 


$$
\bar{F}_{12}=\frac{\mu_{0} I_{1} I_{2}}{4 \pi}\left(\frac{3}{r_{12}^{5}}\left(d \bar{l}_{1}, \bar{r}_{12}\right)\left(d \bar{l}_{2}, \bar{r}_{12}\right)-\frac{2}{r_{12}^{3}}\left(\left(d \bar{l}_{1}, d \bar{l}_{2}\right)\right)\right) \bar{r}_{12}
$$

Here $\boldsymbol{F}_{\boldsymbol{1 2}}$ is a force an element $\boldsymbol{d} \boldsymbol{I}_{\boldsymbol{1}}$ of a conductor 1 with current $\boldsymbol{I}_{\boldsymbol{1}}$ acts on an element $\boldsymbol{d}_{\boldsymbol{2}}$ of a conductor 2 with current $\boldsymbol{I}_{\boldsymbol{2}}$, $\boldsymbol{r}_{\mathbf{1 2}}$ is a vector connecting the centers of conductors elements $\boldsymbol{d l}_{\mathbf{1}}$ and $\boldsymbol{d l}_{\mathbf{2}}$.

This is the formula of interaction by central forces satisfying Newton's third law: the force of action equals the force of counteraction. Ampere's formula explains Ampere's and Faraday's experiments. However, Ampere's formula conflicts with some experiments made by Ampere himself, namely, the action of a constant magnet field on a conductor when the magnetic force is not central: it is orthogonal to the conductor axis.

Disputes continued until 1845 when Grassmann proposed the formula for interaction of conductors elements [9]:

$$
\bar{F}_{12}=\frac{\mu_{0} I_{1} I_{2}}{4 \pi r^{3}}\left[d \bar{l}_{2}\left[d \bar{l}_{1} \bar{r}_{12}\right]\right],
$$

Something different form of this formula is called the Ampere's law:

$$
\bar{F}_{12}=I_{2}\left[d \bar{l}_{2} \bar{B}\right]
$$

where $\boldsymbol{B}$ is induction of the magnetic field of the closed conductor $\boldsymbol{I}_{1}$ circuit

$$
\bar{B}=\frac{\mu_{0} I_{1}}{4 \pi} \oint_{l_{1}} \frac{\left[d \bar{l}_{1} \bar{r}_{12}\right]}{r^{3}}
$$

Grassmann's formula $(2-2)$ is not correct because it violates Newton's third law: the force of action is not equal to the force of counteraction. That is why Ampere's law defines the action of only a closed contour on the element of the conductor, but not vice versa. Tamm explained this: "In the framework of the studying closed constant currents, the force of current elements interaction cannot be determined unambiguously." [3] p.224.

The experiments by Ampere and Faraday were left out of the attention of modern magnetic field theory. It seems that Ampere went beyond the limit of studying the interactions of closed currents. Modern electromagnetism does not go beyond this limit two hundred years later.

\section{Relativity of current-carrying conductors interactions}

A moving element of a neutral conductor with current generates magnetic field $\boldsymbol{H}$ being a superposition of the fields induced by positive and negative charges:

$$
\bar{H}=\frac{1}{c r^{3}}\left(Q^{+}\left[\bar{V}_{1} \bar{r}\right]+Q^{-}\left[\bar{V}_{2} \bar{r}\right]\right) .
$$

Here $\boldsymbol{V}_{\boldsymbol{1}}$ and $\boldsymbol{V}_{\boldsymbol{2}}$ are average velocities of positive and negative charges in the conductor element; $\boldsymbol{Q}^{+}$and $\boldsymbol{Q}^{-}$are, respectively, total positive and negative charges in the conductor. As the total positive and negative charges of any neutral conductor are equal, the above expression can be written as

$$
\bar{H}=\frac{Q^{+}}{c r^{3}}\left[\left(\bar{V}_{1}-\bar{V}_{2}\right) \bar{r}\right] .
$$

The current in the conductor is determined by the difference in velocities of positive and negative charges in the conductor only. In other words, the motion of the conductor with quasi-static speeds does not change its magnetic field. Therefore, the magnetic interaction of two conductor elements is determined by relative motion of the conductors and is independent of the reference frame.

The magnetic force does certain work if the motion of the conductor element is caused by another element generating the force. If the source of the force moves when the first element is in rest, it is relative displacement too. What force does work in this case? It is the force of counteraction ignored by Grassmann.

Let us emphasize the peculiarities of the relativity interaction of conductors elements: forces of action - counteraction are present necessarily and third Newton's law is valid.

We come to the conclusion: motion of a neutral conductor with current caused by its proper magnetic field is impossible because the magnetic field of the conductor does not change in the course of this motion.

What can we say now about Sigalov's experiments? They show the interactions of elements of the same contour. Sigalov was absolutely convinced that Grassmann formula was correct for these interactions, so he did not consider that the forces in these experiments could be axial magnetic forces. By this reason, he concluded that the motion of conductors caused by their proper magnetic field was possible.

Let us show now what interaction model corresponds to the Grassmann expression and why does this expression controvert existence of axial magnetic interactions. 


\section{Magnetic field of conductors with current}

Let's get beyond the limits of the interactions of closed currents and determine why the Grassmann formula for the interaction of conductors elements violates the equality principle of action-counteraction.

Let's write expressions for the forces acting on the elements of two conductors according to the Grassmann formula:

$$
\bar{F}_{12}=\frac{\mu_{0} I_{1} I_{2}}{4 \pi r^{3}}\left[d \bar{l}_{2}\left[d \bar{l}_{1} \bar{r}_{12}\right]\right] ; \quad \bar{F}_{21}=\frac{\mu_{0} I_{1} I_{2}}{4 \pi r^{3}}\left[d \bar{l}_{1}\left[d \bar{l}_{2} \bar{r}_{21}\right]\right] .
$$

Here are the axial vectors, the so-called "pseudo-vectors". The same is the intermediate result for calculating magnetic forces, namely, the vector of magnetic induction $\boldsymbol{B}$ :

$$
\bar{B}_{1}=\frac{\mu_{0} I_{1}}{4 \pi r^{3}}\left[d \bar{l}_{1} \bar{r}_{12}\right] ; \bar{B}_{2}=\frac{\mu_{0} I_{2}}{4 \pi r^{3}}\left[d \bar{l}_{2} \bar{r}_{21}\right] .
$$

The result of using pseudo-vectors is the magnetic force always orthogonal to the axis of the conductor. Therefore, the modern theory cannot explain Ampere's and Faraday's experiments.

Let's turn to the use of polar vectors in magnetic interactions. To do this, we use a known formula

$$
[\bar{a}[\bar{b} \bar{c}]]=\bar{b}(\bar{a}, \bar{c})-\bar{c}(\bar{a}, \bar{b})
$$

We will get

$\bar{F}_{12}=\frac{\mu_{0} I_{1} I_{2}}{4 \pi r^{3}}\left(d \bar{l}_{1}\left(d \bar{l}_{2}, \bar{r}_{12}\right)-\bar{r}_{12}\left(d \bar{l}_{1}, d \bar{l}_{2}\right)\right) ; \bar{F}_{21}=\frac{\mu_{0} I_{1} I_{2}}{4 \pi r^{3}}\left(d \bar{l}_{2}\left(d \bar{l}_{1}, \bar{r}_{21}\right)-\bar{r}_{21}\left(d \bar{l}_{1}, d \bar{l}_{2}\right)\right) . \quad(4-2)$

The result is nontrivial: the magnetic force has two components. However, let us recall Helmholtz's theorem: an arbitrary vector field is the sum of the potential and solenoidal fields.

The second summands in the above formulas, obviously, are the forces of the potential field. They are central, equal in magnitude and opposite in the direction. These forces are the forces of action - counteraction at the same time. The third Newton's law is valid: the force of action is equal to the force of counteraction. These forces are similar to the forces of electric field, namely, the Coulomb's forces, but have the opposite direction.

The first summands, according to the Helmholtz theorem, are the forces of two solenoidal fields. These are two unequal in magnitudes and directions forces. Obviously, they are not the forces of action-counteraction. Interactions by these forces have to match Biot's model: each of these forces causes a counteraction force acting on the force's source.

We can rewrite the expressions (4 - 2) as follows:

$$
\bar{F}_{12}=\bar{U}_{s} I_{2}\left(d \bar{l}_{2}, \bar{r}_{12}\right)+\bar{U}_{p} I_{2}\left(d \bar{l}_{1}, d \bar{l}_{2}\right),
$$

where

$$
\bar{U}_{s}=\frac{\mu_{0} I_{1}}{4 \pi r^{3}} d \bar{l}_{1}, \quad \bar{U}_{p}=-\frac{\mu_{0} I_{1}}{4 \pi r^{3}} \bar{r}_{12} .
$$

We call $\boldsymbol{U}_{\boldsymbol{s}}$ a solenoidal induction of a magnetic field, $\boldsymbol{U}_{\boldsymbol{p}}$ - a potential one.

Let us define the divergence of the potential induction $\boldsymbol{U}_{p}$. The flow of the potential induction vector $\boldsymbol{U}_{\boldsymbol{p}}$ through the surface of the sphere with radius $r$ is equal to

$$
\Phi_{p}=\oint_{s} \frac{-\mu_{0} I_{1}}{4 \pi r^{3}} \bar{r} d s=-\mu_{0} I_{1} .
$$

To determine divergence of the potential induction, let us divide the flow $\boldsymbol{\Phi}_{p}$ by the volume of the sphere:

$$
\operatorname{div} \bar{U}_{p}=-\mu_{0} \rho
$$

where $\rho$ is the volume density of the current in the sphere. The second summands in expressions $(4-2)$ are indeed the forces of a potential field with nonvanishing divergence.

The flow of the induction vector $\boldsymbol{U}_{\boldsymbol{s}}$ of the magnetic field through an arbitrary closed surface is obviously zero due to antisymmetry of the integral. The divergence is zero, so the first summands are indeed components of the magnetic forces of the solenoidal fields. 
In classical electromagnetism, it is considered that the magnetic field is a solenoidal field, its divergence is zero ([3], $\mathrm{p}$. 242). We analyzed the Grassmann formula to have our own conclusions. Why are our conclusions so radically different ones?

We analyzed the interactions of the polar force vectors of the magnetic field, that is, real physical vectors. Classical electrodynamics takes the axial pseudo-vector of the magnetic induction $\boldsymbol{B}$ as the main characteristic of the magnetic field. Therefore, the real properties of the magnetic field are accepted as the properties of the pseudo-vector $\boldsymbol{B}$. The fact that modern electrodynamics considers a magnetic field as a solenoidal field we treat as follows: the field of the magnetic induction vector $\boldsymbol{B}$ is a solenoidal field. The real physical magnetic field has other properties than the field of the magnetic induction vector $B$.

We prove our conclusions to be correct: the real magnetic field consists of two separate fields, the potential and solenoidal ones, acting on moving charges independently. There is no single magnetic field. However, let us return to Ampere's law to understand why the Grassmann formula in this law does not lead to a violation of Newton's third law.

\section{Counteraction forces in Ampere's law}

Let us return to the models of magnetic interactions mentioned in Section 2. The Ampere's interaction model by the central forces corresponds to interactions by forces of a potential field. These interactions are characterized by the central forces and the force of action of one object on the other one is simultaneously the force of counteraction of the second object on the first one. Biot's model is the interaction of the pair of antiparallel forces with equal magnitudes: active non-central force causes the appearance of anti-parallel force of counteraction.

Let us turn to the Grassmann formulas $(4-2)$. Apparently, the potential forces correspond to Ampere's model. Solenoidal components do not respond to the Biot's model because there are no counteraction forces. If our conclusions are correct, why the Grassmann formulas is correct in Ampere's law? Let's look at Ampere's law in polar vectors:

$$
\bar{F}_{12}=\frac{\mu_{0} I_{1} I_{2}}{4 \pi} \oint_{l_{1}} \frac{\left(d \bar{l}_{1}\left(d \bar{l}_{2}, \bar{r}_{12}\right)-\bar{r}_{12}\left(d \bar{l}_{1}, d \bar{l}_{2}\right)\right)}{r^{3}} \quad(5-1)
$$

There are no counteraction forces in this formula. To determine why this formula is correct, we define the formula for the force $\boldsymbol{F}_{21}$ of the conductor's element on the closed circuit. Ampere's law does not violate Newton's third law. To obtain the expression of the force $\boldsymbol{F}_{21}$ having the same magnitude but opposite in the direction, it is enough to change the direction of the vectors $r$ by the opposite one in the last formula

$$
\bar{F}_{21}=\frac{\mu_{0} I_{1} I_{2}}{4 \pi} \oint_{l_{1}} \frac{\left(d \bar{l}_{1}\left(d \bar{l}_{2}, \bar{r}_{21}\right)-\bar{r}_{21}\left(d \bar{l}_{1}, d \bar{l}_{2}\right)\right)}{r^{3}} ; \quad \bar{F}_{21}=-\bar{F}_{12} .
$$

Polar vectors allowed us to obtain the unknown in electromagnetism formula for the action of the conductor's element on a closed circuit. Why in this formula there are no solenoidal components of the conductor elements action on a closed circuit? The reason may be that the total solenoidal force of the conductor's elements on a closed contour is zero:

$$
\bar{F}_{21 s}=\frac{\mu_{0} I_{1} I_{2}}{4 \pi} \oint_{l_{1}} \frac{d \bar{l}_{2}\left(d \bar{l}_{1}, \bar{r}_{12}\right)}{r_{12}^{3}}=0
$$

It was proved in this way [3]. Taking into account that

$$
\frac{\bar{r}_{12}}{r_{12}^{3}}=-\operatorname{grad}\left(\frac{1}{r_{12}}\right)
$$

and the magnitude of this gradient in the direction $\boldsymbol{d l}_{\mathbf{1}}$ equals

$$
\operatorname{grad}_{d \bar{l}_{1}}\left(\frac{1}{r_{12}}\right)=\left(d \bar{l}_{1}, \operatorname{grad}\left(\frac{1}{r_{12}}\right)\right)
$$

we determine the value for the integral of the solenoidal force action by the element $d \boldsymbol{I}_{2}$ on the contour $\boldsymbol{I}_{\mathbf{1}}$ : 


$$
\oint_{l_{1}} \frac{d \bar{l}_{2}\left(d \bar{l}_{1}, \bar{r}_{12}\right)}{r_{12}^{3}}=-d \bar{l}_{2} \oint_{l_{1}}\left(d \bar{l}_{1}, \operatorname{grad}\left(\frac{1}{r_{12}}\right)\right)=-d \bar{l}_{2} \oint_{l_{1}} \frac{\partial\left(\frac{1}{r_{12}}\right)}{\partial l_{1}} d \bar{l}_{1}=0 .
$$

The integral over a closed contour is zero because the integrand is a complete differential.

This means that the total counteraction force by the contour $\boldsymbol{I}_{\boldsymbol{1}}$ on the element of the contour $\boldsymbol{d} \boldsymbol{l}_{2}$ should be zero too. We have identified the reason why Ampere's law is correct in spite of using the Grassmann formula.

Other singularity of the formulas $(5-2)$ is the presence of the solenoidal force by the closed contour $\boldsymbol{I}_{1}$ on element $\boldsymbol{d} \boldsymbol{I}_{2}$ having the same magnitude and opposite direction. This component are counteraction force of the element $\boldsymbol{d l}_{2}$ on the closed contour $\boldsymbol{I}_{\boldsymbol{1}}$. We obtain a confirmation that the action of solenoidal components really corresponds to the Biot's model in Ampere's law: the counteraction force acts on each element of the conductor (source of force) having the same magnitude and opposite direction.

To make the formulas $(4-2)$ correct, i. e., to confirm the Biot's model, we have to add the forces of counteraction to the magnetic forces of the solenoidal field:

$$
\begin{aligned}
\bar{F}_{12}=\frac{\mu_{0} I_{1} I_{2}}{4 \pi r^{3}}( & \left.d \bar{l}_{1}\left(d \bar{l}_{2}, \bar{r}_{12}\right)-\bar{r}_{12}\left(d \bar{l}_{1}, d \bar{l}_{2}\right)-d \bar{l}_{2}\left(d \bar{l}_{1}, \bar{r}_{21}\right)\right) \\
= & \frac{\mu_{0} I_{1} I_{2}}{4 \pi r^{3}}\left(-\bar{r}_{12}\left(d \bar{l}_{1}, d \bar{l}_{2}\right)+d \bar{l}_{1}\left(d \bar{l}_{2}, \bar{r}_{12}\right)+d \bar{l}_{2}\left(d \bar{l}_{1}, \bar{r}_{12}\right)\right) .
\end{aligned}
$$

$$
\bar{F}_{21}=\frac{\mu_{0} I_{1} I_{2}}{4 \pi r^{3}}\left(-\bar{r}_{21}\left(d \bar{l}_{1}, d \bar{l}_{2}\right)+d \bar{l}_{2}\left(d \bar{l}_{1}, \bar{r}_{21}\right)+d \bar{l}_{1}\left(d \bar{l}_{2}, \bar{r}_{21}\right)\right)
$$

We got the correct expressions for the magnetic interactions of conductors elements satisfying the third Newton's law

$$
\bar{F}_{12}=-\bar{F}_{21} \text {. }
$$

In the above expressions, the conductors elements interact by the central forces of the potential components and by pairs of forces action-counteraction of the solenoidal components of the magnetic forces.

Solenoidal force of the counteraction is axial; it is orthogonal to the Grassmann force. The forces of interaction of the conductors elements $\boldsymbol{F}_{\mathbf{1 2}}$ and $\boldsymbol{F}_{\mathbf{2 1}}$ are not orthogonal to the axes of the conductors and may have an arbitrary direction. These forces are antiparallel and have the same magnitude. Fig. 3 shows the magnetic interaction forces of two conductors elements.

Expressions $(5-3)$ indicate that three separate magnetic fields participate in the interaction: two solenoidal fields of the each conductor elements and a common potential field.

The analysis of (4-1) leads us to the conclusion that using the Grassmann formula to describe the interaction of conductors elements is incorrect because it ignores that the potential and the solenoidal forces have different origins. The use of the axial magnetic induction $\boldsymbol{B}$ vector whose norm is the vector sum of potential and solenoidal components,

makes it impossibile to take into account the counteraction forces to solenoidal components of the magnetic field.

Expressions (5-3), obtained by us according to Newton's third law, Whittaker showed in 1910 [10] noting that his formula corresponds to the principle of equality for action-counteraction forces in the conductors elements interaction. However, it is possible to use a shortened version of it corresponding to the Grassmann formula determining the force of the closed contour $\boldsymbol{I}_{\boldsymbol{1}}$ on the element of the contour $\boldsymbol{d}_{\boldsymbol{2}}$. Nevertheless, Whitteker did not emphasize that the Grassmann formula determines the force of the element of conductor on a closed contour incorrectly, violating the principle of equality for action - counteraction forces. 


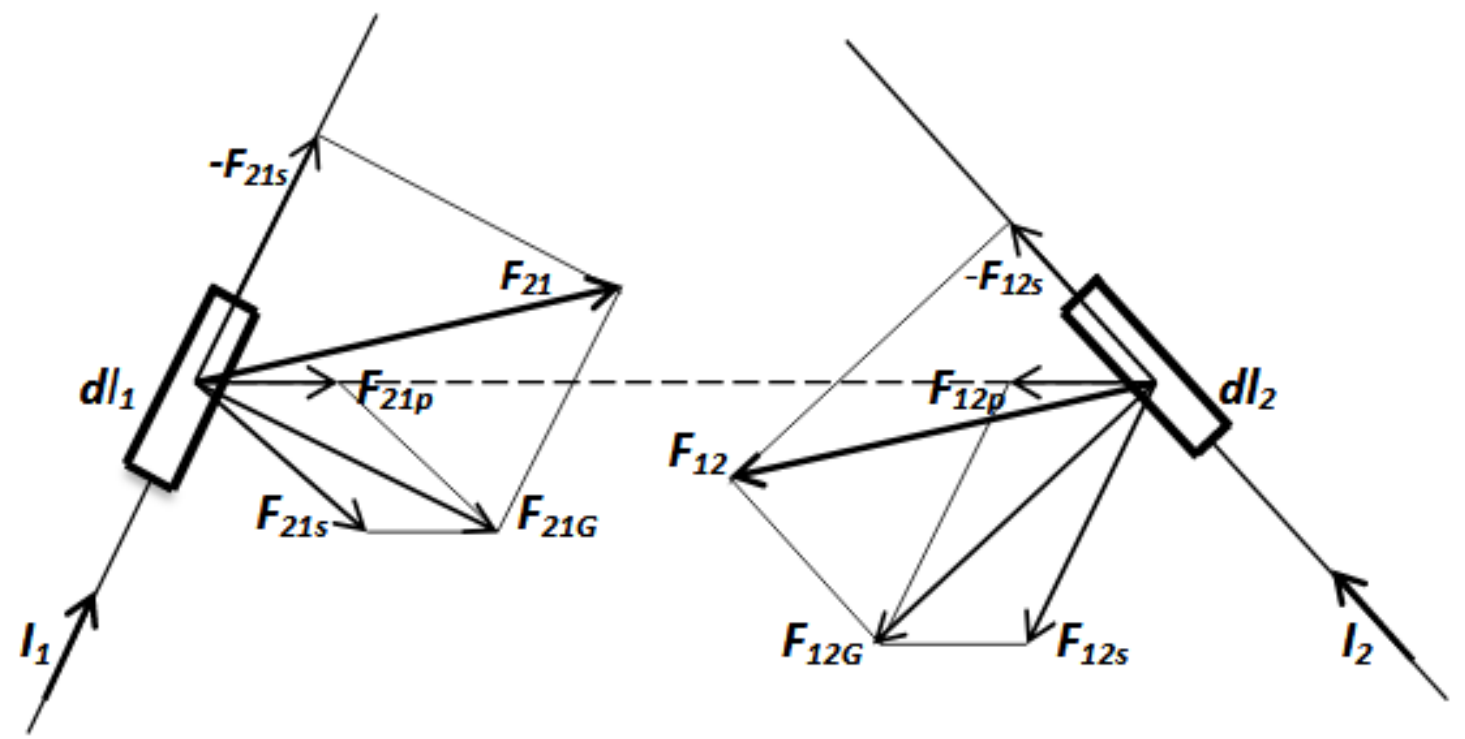

Fig.3. Counteraction forces in the magnetic interaction of conductors. Here $F_{12 G}$ and $F_{21 G}$ are the Grassmann forces, $\quad-F_{12 s}$ and $-F_{21 s}$ are counteraction forces.

Let us write Ampere's law formulas for various cases.

1. Interaction of elements of conductors - Whittaker's formula:

$$
\begin{gathered}
\bar{F}_{12}=\frac{\mu_{0} I_{1} I_{2}}{4 \pi r^{3}}\left(-\bar{r}_{12}\left(d \bar{l}_{1}, d \bar{l}_{2}\right)+d \bar{l}_{1}\left(d \bar{l}_{2}, \bar{r}_{12}\right)+d \bar{l}_{2}\left(d \bar{l}_{1}, \bar{r}_{12}\right)\right) \\
=\frac{\mu_{0} I_{1} I_{2}}{4 \pi r^{3}}\left(\left[d \bar{l}_{2}\left[d \bar{l}_{1} \bar{r}_{12}\right]\right]+d \bar{l}_{2}\left(d \bar{l}_{1}, \bar{r}_{12}\right)\right) .
\end{gathered}
$$

The formula correctly determines interaction of elements of conductors, so it may be used in all cases of interaction. One should use it while determining interaction forces for parts of conductors of the same loop. Whittaker's formula can be simplified if a closed contour(s) takes part in interaction.

2. Action of closed contour $I_{1}$ on conductor 2 element (the Grassmann formula, Ampere's law):

$$
\bar{F}_{12}=\frac{\mu_{0} I_{1} I_{2}}{4 \pi} \oint_{l_{1}} \frac{-\bar{r}_{12}\left(d \bar{l}_{1}, d \bar{l}_{2}\right)+d \bar{l}_{1}\left(d \bar{l}_{2}, \bar{r}_{12}\right)}{r_{12}^{3}}=\frac{\mu_{0} I_{1} I_{2}}{4 \pi} \oint_{l_{1}} \frac{\left[d \bar{l}_{2}\left[d \bar{l}_{1} \bar{r}_{12}\right]\right]}{r_{12}^{3}} \text {. }
$$

3. Action of conductor $\boldsymbol{I}_{1}$ element on closed contour $\boldsymbol{I}_{2}$ :

$$
\bar{F}_{12}=\frac{\mu_{0} I_{1} I_{2}}{4 \pi} \oint_{l_{2}} \frac{-\bar{r}_{12}\left(d \bar{l}_{1}, d \bar{l}_{2}\right)+d \bar{l}_{2}\left(d \bar{l}_{1}, \bar{r}_{12}\right)}{r_{12}^{3}} .
$$

We have to note: this formula is not the Grassmann formula, because its second component is the force of counteraction caused by the force of action of contour element $\boldsymbol{d l}_{\mathbf{2}}$ on element $\boldsymbol{d \boldsymbol { l } _ { \boldsymbol { 1 } }}$.

4. Interaction of closed contours $\boldsymbol{I}_{1}$ and $\boldsymbol{I}_{2}$ :

$$
\bar{F}_{12}=\frac{\mu_{0} I_{1} I_{2}}{4 \pi} \oint_{l_{1}} \oint_{l_{2}} \frac{-\bar{r}_{12}\left(d \bar{l}_{1}, d \bar{l}_{2}\right)}{r_{12}^{3}}
$$

The interaction of closed circuits is performed exclusively by the potential components of the magnetic forces. This contradicts the conclusion of classical electrodynamics: the magnetic field is a solenoidal one. 


\section{Axial magnetic interactions of elements of conductors}

Let us verify (5-3) on the basis experiments of Ampere and Faraday that proved the existence of axial magnetic forces. Consider the interaction of the $\boldsymbol{d}_{\boldsymbol{1}}$ element of a rectilinear conductor with current $\boldsymbol{I}_{\boldsymbol{1}}$ with element $\boldsymbol{d}_{\boldsymbol{2}}$ which is the continuation of this conductor in mercury (Fig. 4). Let $A O$ be a metal part of the conductor and $O B$ - its part in mercury.

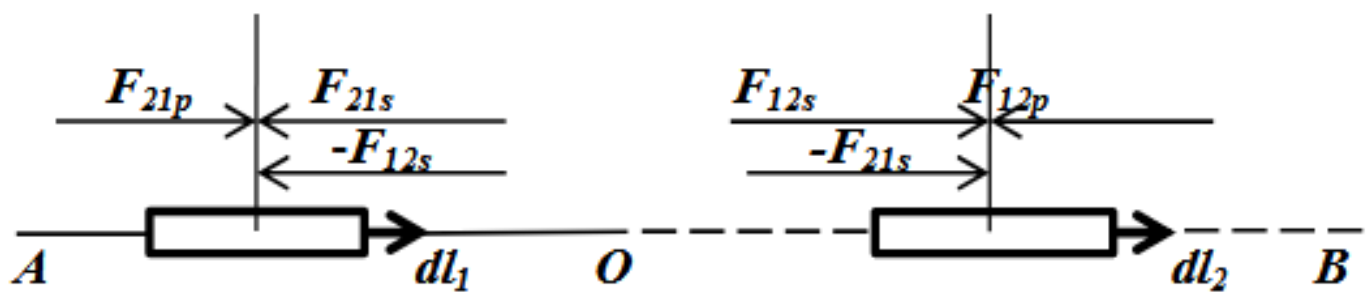

Fig. 4. Interaction between element $d l_{1}$ of the conductor with current $l_{1}$ and element $d l_{2}$ of the part of this conductor in mercury

First, we determine forces between elements of conductors using the Grassmann formulas (4 - 2):

$$
\bar{F}_{12}=\frac{\mu_{0} I^{2}}{4 \pi r^{3}}\left(-\bar{r}_{12}\left(d \bar{l}_{1}, d \bar{l}_{2}\right)+d \bar{l}_{1}\left(d \bar{l}_{2}, \bar{r}_{12}\right)\right) \text {. }
$$

Potential and solenoidal components of forces have the same magnitude and balance each other as their directions are opposite. Biot-Savart law describes the total action of potential and non-potential components of magnetic forces. As a result, the magnetic induction in axial direction of the conductor is zero. Hence, the total acting force is zero, then what the counteraction force may arise in this case? If the segregation of two different components of the magnetic force is mathematical abstraction only, the action force along the axial direction should be zero. However, the experiments by Faraday, Ampere et al. deny it. Perhaps the point is that the interaction in these experiments takes place in mercury and their results is the consequence of electrolytic reactions?

Let us consider the device by Nikolayev [11]: the rotor is a ring shaped conductor with current $\boldsymbol{I}$ rotating in the magnetic field of two semi-circular solenoids (Fig. 5). Current is supplied to the rotor through sliding metal terminals.

The ring shaped conductor (motor's rotor) can be rotated by the axial magnetic forces only. This example proves that axial forces exist in case of metal contacts too.

Let's return to the consideration of the interaction in Fig. 4. Let's use the Whittacker's formula

$$
\bar{F}_{12}=\frac{\mu_{0} I_{1} I_{2}}{4 \pi r^{3}}\left(-\bar{r}_{12}\left(d \bar{l}_{1}, d \bar{l}_{2}\right)+d \bar{l}_{1}\left(d \bar{l}_{2}, \bar{r}_{12}\right)+d \bar{l}_{2}\left(d \bar{l}_{1}, \bar{r}_{12}\right)\right) \text {. }
$$

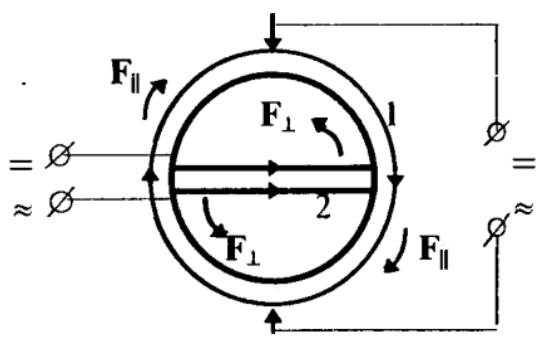

Fig.5. Nikolayev's motor

Three forces of the same magnitude act on every element of the conductor along the axial direction: potential component, solenoidal component, and the counteraction force induced by the solenoidal component of the action of the second element on the first one. Potential and solenoidal components balance each other, but the counteraction force is not balanced, this is the force of repulsion (the last component in the formula above).

The result contradicts the theory: the repulsion force acts on conductors elements with the same direction of currents. The acting force is the force of counteraction to the solenoidal component of the magnetic field.

We get a similar result having determined the force of the element $\boldsymbol{d l}_{2}$ on the element $\boldsymbol{d l}_{\boldsymbol{1}}$ : the elements of the conductors are repulsed when the directions of the currents coincide. Changing the direction of the current does not change the directions of the magnetic forces. We confirmed the above experiments by Faraday and Ampere. The Whittaker's formula describes these experiments correctly. We add: when the currents in the conductors elements are opposite, they are attracted. 
The analysis of the above experiments proves: the potential and solenoidal components of the magnetic forces are real and act independently. Therefore, the solenoidal component balanced by the potential component induces counteraction force along the axial direction.

To have an idea on the magnitude order of these forces, let us determine the force of tension appearing in the conductor when current passing through it equals, say, one thousand Amperes. Let us write the expression for the magnetic force in the transversal section of the straight conductor. We determine the force with which a direct conductor of $1 \mathrm{~m}$ in length with a current acts on its continuation of the same length. We have to determine the minimum distance between the conductor and its elongation because for zero value we have infinity. We calculate minimum distance basing on

concentration $\boldsymbol{n}_{\boldsymbol{0}}$ of electrons in a copper conductor

$$
n_{0}=k \frac{N_{A} D}{A}, \quad n_{0}=2 \frac{6.02 \times 10^{23} \times 8.96 \times 10^{6}}{63.546}=0.16976 \times 10^{30} \mathrm{M}^{-3} .
$$

where $\boldsymbol{k}$ is metal valence, $\boldsymbol{N}_{\boldsymbol{A}}$ - Avogadro's number, $\boldsymbol{D}$ - density of metal, $\boldsymbol{A}$ - atomic weight/mass.

Basing on the concentration value, we determine the average distance a between electrons:

$$
a=\frac{1}{\sqrt[3]{n_{0}}}=1.806 \times 10^{-10} \mathrm{M}
$$

Let us write expression for the magnetic force:

$$
\bar{F}_{12}=\frac{\mu_{0} I^{2}}{4 \pi} \int_{x_{2}=-b}^{x_{2}=0} \int_{x_{1}=a}^{x_{1}=b} \frac{d x_{2} d x_{1}}{\left(x_{1}-x_{2}\right)^{2}},
$$

where from $\boldsymbol{x}=\boldsymbol{a}$ to $\boldsymbol{x}=\boldsymbol{b}$ is the segment of the conductor $\mathbf{1}$, from $\boldsymbol{x}=\mathbf{0}$ to $\boldsymbol{x}=-\boldsymbol{b}$ is the segment of its elongation (conductor 2). From $x=0$ to $x=a$ is the distance between the conditional end of the conductor and the beginning of its elongation.

We take into account some elementary formulas:

$$
\int \frac{d x}{(x-c)^{2}}=\frac{-1}{x-c} ; \int \frac{d x}{c-x}=-\ln (c-x) ; \int \frac{d x}{x-c}=\ln (x-c) .
$$

Expression for force $\boldsymbol{F}_{12}$ looks like

$$
\begin{aligned}
\bar{F}_{12}=\frac{\mu_{0} I^{2}}{4 \pi} \int_{x_{2}}=-b & \int_{x_{1}=a}^{x_{2}}=0 \\
& =\frac{\mu_{0} I^{2}}{4 \pi}((\ln b-\ln 2 b)-(\ln a-\ln b))=\frac{\mu_{0} I^{2}}{4 \pi}(-\ln a+2 \ln b-\ln 2 b) .
\end{aligned}
$$

If current equals $1000 \mathrm{~A}$ and the conductor is $1 \mathrm{~m}$ long the force equals $2.3128 \mathrm{~N}$. If a conductor is $10 \mathrm{~m}$ long then the force equals $2.4044 \mathrm{~N}$.

\section{Biot-Savart's law}

The Biot-Savart's law in electrodynamics is interpreted as denying the possibility of the axial interaction between conductors elements and moving charges. But it is necessary to take into account the significant shortcomings of this law. The law defines the properties of the axial pseudo-vector of magnetic induction $\boldsymbol{B}$, which is a function of the sum of the potential and solenoidal components of the magnetic field. Therefore, this law takes into account the actions of only active magnetic forces and neglects the forces of counteraction.

We emphasize the essential difference between the Coulomb and the "anti-Coulomb" forces. The module of Coulomb's force does not depend on the velocity of charges as the objects of interaction, the module of "anti-Coulomb" force is a function of the velocities of these charges.

Let us try to understand the principle of $\mathrm{E}-\mathrm{H}$ aerials operation mentioned in the Introduction. Consider the action of the potential and solenoidal components of the magnetic forces. Potential component is the force of attraction. It's module does not depend on the vector $\boldsymbol{r}$ direction. The solenoidal component is the axial repulsion force with the module proportional to the projection of the conductor element onto vector $r$. Module of this force is zero in the orthogonal direction to the axis of the conductor. It is equal to the potential component in the axial direction but acts in opposite direction. So, along the axis, the sum of both components is zero. The original communication test [12] used two regular serial USW radio stations "Berkut 603" withen the emitting and receiving antennas oriented along a straight line.

The receiving antenna coil is screened by the copper cylindrical screen. Antenna passes through two holes located on the axis of the cylindrical screen. The reception of a radio signal in the axial direction of the emitting antenna confirms 
the axis of the cylindrical screen. The reception of a radio signal in the axial direction of the emitting antenna confirms conclusions on the existence of two components of the magnetic field. The antenna emits two components of the magnetic field in axial direction. They counterbalance each other acting in an antiphase. The cylindrical copper screen, where the receiving antenna coil is located, can be treated as a closed conductor orthogonal to the axis of the antenna absorbing magnetic field potential component. The receiving antenna coil receives a solenoidal component only.

The use of $\mathrm{E}-\mathrm{H}$ antennas has fundamental interest demonstrating the possibility to isolate and use the solenoidal component of the magnetic field along the axial direction of the conductor.

\section{Rotating moments in magnetic interactions}

Solenoidal components of magnetic action and counteraction forces do not act along the same straight line. This indicates that the magnetic interaction of conductors elements contains rotating moments

$$
\bar{M}_{12}=\left[\bar{r}_{12}\left(\bar{F}_{12 s}-\bar{F}_{21 s}\right)\right] ; \quad \bar{M}_{21}=\left[\bar{r}_{21}\left(\bar{F}_{21 s}-\bar{F}_{12 s}\right)\right] ; \quad \bar{M}_{12}=\bar{M}_{21} \text {. }
$$

It looks strange that the rotating moments have the same direction. Does it reflect the reality? Sigalov [7] gives his version to Faraday experiment on magnet rotation floating in electrolyte around the fixed conductor (Fig. 6). Moreover, Sigalov made another experiment on rotation of a conductor around a fixed magnet. These experiments show that the magnet rotates around the conductor and the conductor rotates around the magnet in the same direction if the current direction is the same. Nikolayev's experiments [11] also proved that magnet and conductor rotations have the same direction (Fig. 7). Therefore, their experiments show that the rotating moments in magnetic interactions really have the same direction. It means that the interaction of the conductor and the magnet is not an ordinary mechanical interaction transfer of rotary motion from one objects to another. The solenoidal component of the magnetic field generates a vortex rotating two interacting objects in the same direction. Solenoidal component of the magnetic field is a real third participant of such interaction - the solenoidal magnetic field really rotates. The Oersted model for the vortex nature of the magnetic interaction is confirmed.

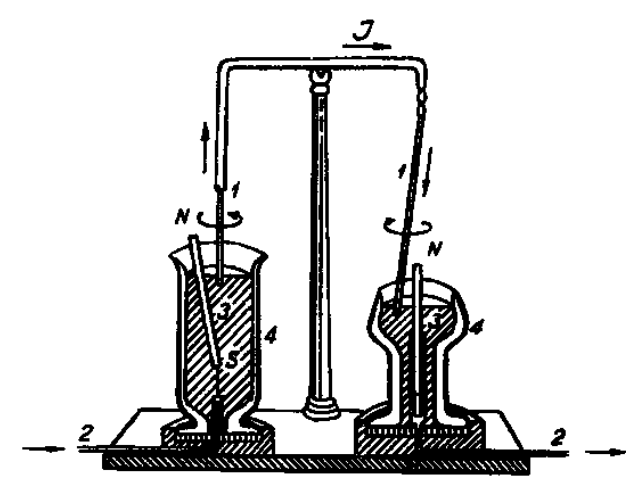

Fig.6. Sigalov's experiments
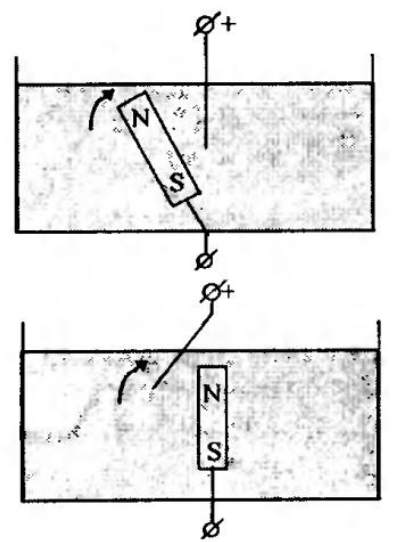

Fig. 7. Nikolayev's experiments

\section{Magnetic component of Lorentz force}

Magnetic component of Lorentz force is a magnetic force acting on a charge moving in the magnetic field. When induction $\boldsymbol{B}$ of the magnetic field is generated by a closed contour, Lorentz force formula determines it correctly:

$$
\bar{F}=[\bar{V} \bar{B}] \text {. }
$$

However, the same formula applies to interaction of moving charges:

$$
\bar{F}_{12}=\frac{\mu_{0} q_{1} q_{2}}{4 \pi r^{3}}\left[\bar{V}_{2}\left[\bar{V}_{1} \bar{r}_{12}\right]\right]
$$

where $\boldsymbol{q}_{1}, \boldsymbol{q}_{2}$ are charges moving with velocities $\boldsymbol{V}_{1}, \boldsymbol{V}_{2}$, respectively.

When magnetic field is generated by another moving charge, we must consider the counteraction force too, and the formula similar to Whittaker's formula will be correct in this case:

$$
\bar{F}_{12}=\frac{\mu_{0} q_{1} q_{2}}{4 \pi r^{3}}\left(-\bar{r}_{12}\left(\bar{V}_{1}, \bar{V}_{2}\right)+\bar{V}_{1}\left(\bar{V}_{2}, \bar{r}_{12}\right)+\bar{V}_{2}\left(\bar{V}_{1}, \bar{r}_{12}\right)\right)
$$

Magnetic field does not change particle energy when the magnetic force is orthogonal to the direction of its motion. However, magnetic force may have any direction in the case of magnetic interaction of charges, and this leads to a change in the energy of particles. When particles move in parallel in the axial direction, the magnetic force is repulsive one. It is the same model of interaction analyzed earlier for the conductors elements. When the particles move antiparallelly in the axial direction, the magnetic force is attractive one.

\section{Conclusion}


The magnetic field in the classical electrodynamics is axial pseudo-vector of magnetic induction $\boldsymbol{B}$. This field is virtual and artificial. It does not exist in nature. Its properties are not the properties of a real magnetic field. One example is the definition of magnetic field as a solenoidal one. The use of pseudo-vectors leads to the errors in expressions for the magnetic interaction of conductors elements and the interaction of moving charges, i.e., a violation of the equality principle for the forces of the action and counteraction.

we use real physical polar vectors to describe the magnetic field. This enables us to prove that a real magnetic field has

two components existing and acting independently: a potential and a solenoidal ones. The article presents the correct expression for the differential form of the Ampere's law and the correct interpretation of the Biot-Savart's law, which does not deny axial interactions and explains most of the "paradoxical" experiments in electrodynamics.

\section{ACKNOLEDGMENTS}

The author thanks Dr Wolodymyr Kozyrski at the Bogolubov Institute for Theoretical Physics (Kiev) for encouragement and fruitful discussions.

\section{REFERENCES}

1. Petuschak, V., Kozlova, N. and Osyka, B. 2014 Ampere's law and paradoxical experiments in electrodynamics, Electronics and information technologies. Issue 4. P. 174-175.

2. Ampère, A. 1826 (in German), Théorie des phénomènes électro-dynamiques, uniquement déduite de l'expérience, Méquignon-Marvis. Retrieved 2010-9-26.

3. Tamm, I. 1979 Fundamentals of the theory of electricity - Mir publisher, Moscow

4. Ørsted, H. C. 1820 (in latin) Experimenta circa effectum conflictus electrici in acum magneticam, Typis Schultzinis.

5. Faraday, M. 1846 Experimental researches of electricity Phil. Trans. R. Soc. Lond. 1846 136, 1-20

6. Maxwell, J. S. 1873 A Treatise on Electricity and Magnetism vol. 2 Oxford Clarendon press.

7. Sigalov, R. G. et al.1965 (in Russian) New investigations of magnetomotive forces. (Science Publishing, Tashkent, Uzbekistan)

8. Pappas, P. T. 1983 The Original Ampere Force and Biot-Savart and Lorentz Forces . II Nuovo Cimento. 11 Agosto, 1983,V.76 B, №2, P 189-196.

9. Grassman, H. 1845 Neue Theorie der Elektrodynamik. Annalen der Physik und Chemie - Bd. 64, №1- S. $1-18$.

10. Whittaker, E. 1910 A history of the theories of aether and electricity. The classical theories. Thomas Nelson and Sons Ltd, London.

11. Nikolaev, G. V. 1997 (in Russian) Consistent electrodynamics. Theory, experiments, paradoxes. STL Publishing, Tomsk, Russia.

12. Korobeynikov,V. 2005 (in Russian) A new kind of electromagnetic radiation. http://n-t.ru/tp/ts/nv.htm

\section{Author' biography}

I, Valery Petuschak, was born on December 25, 1939 in Donetsk, Ukraine, Soviet Union. I studied in Polytechnic institute in Kiev 1958 -1963 years, got a profession of an electrical engineer. I worked at the Institute of Cybernetics of the Academy of Sciences of Ukraine from 1962 to 2000 at the department of Theory of Digital Machines, first as an engineer, since 1974, as a research associate. I received PhD degree in 1974.

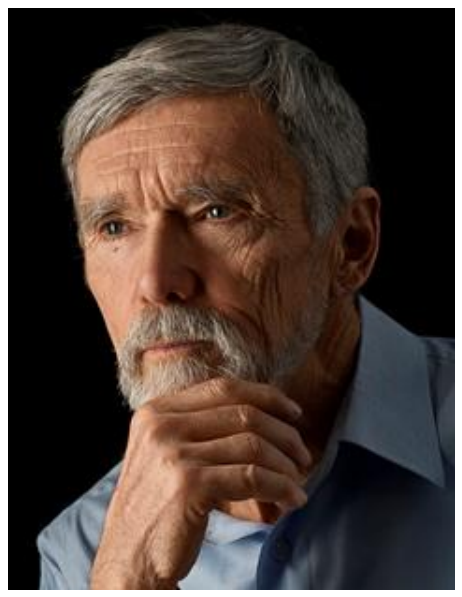

